\title{
Maternal prepregnancy body mass index and risk of bronchopulmonary dysplasia
}

\author{
Suzan L. Carmichael ${ }^{1}$, Peiyi Kan ${ }^{1}$, Jeffrey B. Gould ${ }^{1}$, David K. Stevenson ${ }^{1}$, Gary M. Shaw ${ }^{1}$ and Henry C. Lee ${ }^{1}$
}

BACKGROUND: We examined the relationship between women's prepregnancy BMI and development of bronchopulmonary dysplasia (BPD) in their preterm offspring, hypothesizing that obesity-associated inflammation may increase risk.

METHODS: We studied infants born in California between 2007 and 2011, using linked data from California Perinatal Quality Care Collaborative neonatal intensive care units, hospital discharge, and vital statistics. We included infants with birthweight $<1,500 \mathrm{~g}$ or gestational age at birth of 2229 weeks. BPD was defined as continuous supplemental oxygen use at 36 weeks' postmenstrual age.

RESULTS: Among 12,621 infants, 4,078 (32\%) had BPD. After adjustment for maternal race/ethnicity, age, education, payer source, and infant sex, BMI status underweight I (BMI < 16.9, odds ratio (OR) 1.7, 95\% confidence interval (CI) 1.3-2.1) and obesity III (BMI $\geqslant 40.0$, OR 1.3, 95\% Cl 1.0-1.6) were associated with an increased risk of BPD. When considering maternal BMI as a continuous variable, a nonlinear association with BPD was observed for male infants and infants delivered at 25-29 weeks of gestational age, but not for other subgroups.

CONCLUSION: Both high and low maternal BMI were associated with increased BPD risk. These findings support the notion that BPD is a multi-factorial disease that may sometimes have its origins in utero and be influenced by maternal inflammation.

B ronchopulmonary dysplasia (BPD), or chronic lung disease of the neonate, is a disease of preterm infants, characterized by airway injury, inflammation, and prolonged need for oxygen and ventilator therapy (1-3). It predominantly occurs among infants born very early or very low birthweight (VLBW), with the majority of cases occurring among infants born at $<28$ weeks of gestation or $<1,500 \mathrm{~g}$ $(1,4)$. Although there are known risk factors for BPD, such as lower birthweight, younger gestational age at birth, and male sex, much of the risk profile is still unexplained (5). The "classic" form of BPD primarily due to trauma caused by mechanical ventilation described by Northway and colleagues has become less frequent (2). However, there is an increasing number of preterm infants who develop a different form of lung injury of so-called "new BPD," which may be more attributable to inflammatory processes both in utero and after birth, leading to longer hospitalization and potential long-term adverse outcomes (2,4,6-8). Known contributing proinflammatory exposures include, for example, chorioamnionitis, neonatal sepsis, and oxygen exposure via ventilation $(6,7,9-11)$.

Maternal obesity is also a pro-inflammatory exposure. It increases the risk of a variety of adverse maternal and neonatal outcomes, such as preterm delivery, especially very early preterm delivery (e.g., <30 weeks), preeclampsia, postpartum hemorrhage, and neonatal hypoglycemia (12-16). Research suggests that it predisposes offspring to an increased risk of adverse outcomes during childhood as well, such as insulin resistance, high blood pressure, and respiratory illnesses such as asthma $(17,18)$. Many mechanisms have been proposed to contribute to these associations, including alterations in maternal and offspring immune and inflammatory responses (17). Although less frequently studied, maternal underweight BMI is also associated with an increased risk of preterm birth and other adverse outcomes (19).

Our hypothesis is that extremes of maternal BMI are associated with compromises in the in utero environment, such as an altered inflammatory response, which in turn may increase the vulnerability of at-risk newborns to develop BPD, a condition that is mediated partly by in utero inflammation. To address this hypothesis, we examined whether maternal prepregnancy BMI was associated with the risk of development of BPD among infants born at $<30$ weeks gestation, using data on California births from 2007 to 2011.

\section{METHODS \\ The California Perinatal Quality Care Collaborative (CPQCC) collects data from 136 neonatal intensive care units (NICUs) in California, representing more than $95 \%$ of very low birthweight $(<1,500 \mathrm{~g})$ infant admissions across the state (20). CPQCC data collection is based on an expanded version of the Vermont Oxford Network data set, which has developed standard definitions of morbidities in NICU patients (21). CPQCC and the Vermont Oxford Network are interdisciplinary networks of practice engaging in}




\section{Maternal BMI and BPD Articles}

improving care for patients in NICUs by using data collected at each unit in order to allow for comparisons of outcomes over time, with other centers, and following quality improvement activities. Member NICUs collect data on their patients in a prospective manner, with each record undergoing logic and missing data checks. For the current analysis, CPQCC data were linked to data from the Office of Statewide Health Planning and Development (OSHPD), which consolidates all of California's hospital discharge data and vital statistics data. The rationale for linking these data sets is that while CPQCC collects detailed clinical data that would be more accurate than administrative data for conditions such as BPD, it does not include certain maternal data such as BMI, which are available in OSHPD data files. In addition to other eligibility criteria, CPQCC collects data on all infants in member NICUs who had very low birthweight or were born at 22-29 weeks of gestation. We limited our analysis to patients who were inborn or transferred to the hospital within 2 days of birth and 22-29 weeks of gestation at delivery. Using these criteria, greater than $99 \%$ of eligible CPQCC records were linked to OSHPD data.

We examined all births that occurred from 1 January 2007 to 31 December 2011 - the most recent years for which data were available. BPD was defined as any infant requiring continuous supplemental oxygen at 36 weeks' postmenstrual age or at discharge to home, whichever occurred earlier; these data were collected by CPQCC and are based on the definitions developed by the Vermont Oxford Network. We excluded infants who died before 36 weeks' postmenstrual age; had they survived, their risk of development of BPD may have been relatively high, but is ultimately uncertain. Gestational age was the obstetric estimate of gestation at delivery in weeks provided on the birth certificate. BMI was defined as selfreported maternal weight $(\mathrm{kg})$ divided by height-squared $\left(\mathrm{m}^{2}\right)$, as reported on the birth certificate. Other covariates included maternal race/ethnicity, age, education, principal source of payment for delivery, and infant sex $(5,22)$.

There were 2,580,178 live-born infants from 2007 to 2011. Of these, 19,560 infants had a gestational age of 22-29 weeks, with 18,828 having birthweight that was consistent with the published norms for gestational age (we excluded those with birthweights for gestational age that were below the 1st or greater than the 99th percentiles) (23). Of those records, we excluded 1,460 infants who were not linked to CPQCC data, 2,015 infants who had major congenital malformations as per CPQCC definitions (24), 81 outborn infants who were transferred to the hospital after 2 days of life, and 2,651 additional infants who died before 36 weeks' postmenstrual age, leaving 12,621 births eligible for analysis. Among these infants, 4,078 had BPD, 3,941 were on oxygen at 36 weeks' postmenstrual age, and 137 were on oxygen at hospital discharge at $<36$ weeks. A total of 7,863 infants did not have BPD and did not die before 36 weeks' postmenstrual age and were considered as "controls", and 680 infants were excluded because of unknown BPD status. After excluding case and control infants with missing data on any covariates and three subjects with maternal $\mathrm{BMI}>75$, there were 3,456 BPD cases who survived to at least 36 weeks' postmenstrual age and 6,726 controls available for analysis. A total of $76.3 \%$ of cases and $74.6 \%$ of controls were singletons.

To estimate risks, we conducted logistic regression analyses that included the covariates mentioned above. We first examined BMI as a categorical variable, based on NIH/NHLBI guidelines (http://www. nhlbi.nih.gov/health/public/heart/obesity/lose_wt/bmi_dis.htm). We then examined models that included BMI as a continuous variable, as a test for linearity of association, and then added BMI-squared, as a test for nonlinearity of association. This approach avoids misclassification from categorization and makes full use of all actual values of the data. These results were examined for all cases together, as well as separately, for male and female infants, for infants born at 22-24 vs. 25-29 weeks of gestation, and by race/ethnicity. We also examined results after excluding mothers with prepregnancy or pregnancyassociated hypertension or prepregnancy or gestational diabetes.
Table 1. Descriptive characteristics of infants with $(n=3,456)$ and without $(n=6,726)$ bronchopulmonary dysplasia

\begin{tabular}{lcc}
\hline & $\begin{array}{c}\text { No. of BPD cases } \\
(\%)\end{array}$ & $\begin{array}{c}\text { No. of non-cases } \\
(\%)\end{array}$ \\
\hline $\begin{array}{l}\text { Maternal race/ethnicity } \\
\text { Non-Hispanic White }\end{array}$ & $855(24.7)$ & $1,710(25.4)$ \\
US-born Hispanic & $897(26.0)$ & $1,707(25.4)$ \\
Foreign-born Hispanic & $878(25.4)$ & $1,587(23.6)$ \\
African-American & $438(12.7)$ & $911(13.5)$ \\
Asian & $240(6.9)$ & $520(7.7)$ \\
Other & $148(4.3)$ & $291(4.3)$
\end{tabular}

$\begin{array}{llr}\begin{array}{l}\text { Maternal age (years) } \\ <20\end{array} & 380(11.0) & 689(10.2) \\ 20-24 & 678(19.6) & 1,237(18.4) \\ 25-29 & 800(23.1) & 1,547(23.0) \\ 30-34 & 823(23.8) & 1,681(25.0) \\ 35-39 & 566(16.4) & 1,172(17.4) \\ 40+ & 209(6.0) & 400(5.9)\end{array}$

\section{Maternal education}

Less than High Schoo

$1,630(24.2)$ GED

High School graduate or

$973(28.2)$

$1,822(27.1)$

Some college

$684(19.8)$

1,397 (20.8)

Associate degree

$221(6.4)$

$334(5.0)$

Undergraduate degree

$502(14.5)$

$996(14.8)$

Postgraduate degree

$243(7.0)$

$547(8.1)$

Principal source of payment for delivery

$\begin{array}{lcc}\text { Medi-Cal } & 1,678(48.6) & 3,199(47.6) \\ \text { Private } & 1,576(45.6) & 3,119(46.4) \\ \text { Uninsured } & 96(2.8) & 203(3.0) \\ \text { Other } & 106(3.1) & 205(3.0)\end{array}$

Gestational age at delivery

$\begin{array}{lcr}22 \text { Weeks } & 36(1.0) & 12(0.2) \\ 23 \text { Weeks } & 209(6.1) & 77(1.1) \\ 24 \text { Weeks } & 563(16.3) & 258(3.8) \\ 25 \text { Weeks } & 673(19.5) & 526(7.8) \\ 26 \text { Weeks } & 598(17.3) & 757(11.3) \\ 27 \text { Weeks } & 568(16.4) & 1,176(17.5) \\ 28 \text { Weeks } & 458(13.3) & 1,697(25.2) \\ 29 \text { Weeks } & 351(10.2) & 2,223(33.1)\end{array}$

Infant sex

$\begin{array}{lll}\text { Female } & 1,458(42.2) & 3,259(48.5) \\ \text { Male } & 1,998(57.8) & 3,467(51.5)\end{array}$

BPD, bronchopulmonary dysplasia. 
Table 2. Association of risk of bronchopulmonary dysplasia with maternal prepregnancy BMl, specified as a categorical variable, California, 2007-2011 ${ }^{\mathrm{a}}$

\begin{tabular}{|c|c|c|c|}
\hline BMI category $\left(\mathrm{kg} / \mathrm{m}^{2}\right)$ & No. of BPD cases (\%) & No. of non-cases (\%) & OR $(95 \% \mathrm{Cl})$ \\
\hline Underweight II/III (moderate to severe thinness) $(<16.9)$ & $28(0.8)$ & $57(0.8)$ & $1.19(0.73-1.94)$ \\
\hline Underweight I (mild thinness) (17.0-18.4) & $129(3.7)$ & $194(2.9)$ & $1.65(1.28-2.12)$ \\
\hline Overweight (25.0-29.9) & $936(27.1)$ & $1,735(25.8)$ & $1.07(0.96-1.20)$ \\
\hline Obesity I (30.0-34.9) & $547(15.8)$ & $1,023(15.2)$ & $1.04(0.91-1.19)$ \\
\hline
\end{tabular}

${ }^{a}$ Analyses included 3,456 BPD cases and 6,726 non-cases and were adjusted for maternal race/ethnicity, age, education, and principal source of payment for delivery, and gestational age and infant sex.

Approval to conduct these analyses was obtained from the California Committee for the Protection of Human Subjects.

\section{RESULTS}

Characteristics of infants with and without BPD are shown in Table 1. Younger gestational age and male sex were more frequent among BPD cases. Maternal race/ethnicity, age, education, and principal payment source were similar for cases and controls.

The association of categories of maternal prepregnancy BMI and risk of BPD is shown in Table 2. After covariate adjustment, offspring of women with underweight $I$ and obesity III status had an increased risk of BPD; the respective odds ratios (ORs) were 1.7 (95\% confidence interval (CI) 1.32.1 ) and 1.3 (95\% CI 1.01-1.6). The OR for underweight II/III women was $1.2(0.7-1.9)$, but estimation in this group was limited by small sample size ( 28 cases).

Results from analyzing BMI as a continuous variable did not support a linear association of BMI with risk of BPD $(P$-values for $\mathrm{BMI}$ as a continuous term ranged from 0.56 to 1.00 , in models that did not include a quadratic term, or BMI-squared). Results did support a nonlinear association, for all cases together, male infants, and infants delivered at 25-29 weeks of gestation $(P$-values for BMI-squared terms were all $<0.01)$, but not for female infants $(P=0.11)$ or infants delivered at 22 24 weeks $(P=0.53)$. The models suggested that risks were elevated at both extremes of BMI (Figure 1). For example, among all infants, the ORs for the 1st percentile and 99th percentiles of BMI were 1.2 (95\% CI 1.1-1.4) and 1.4 (95\% CI 1.1-1.7), among male infants the respective ORs were 1.3 (95\% CI 1.1-1.5) and 1.5 (95\% CI 1.1-2.1), and among births at 2529 weeks they were $1.2(1.1-1.4)$ and $1.4(1.1-1.8)$ (Figure 1).

When examining racial/ethnic groups separately, nonHispanic whites were the only group for which results provided statistical evidence for a nonlinear association with BMI (BMI-squared $P<0.01$ ); the ORs for the 1st and 99th percentiles of BMI (i.e., 17.3 and $\left.47.6 \mathrm{~kg} / \mathrm{m}^{2}\right)$ were $1.4(95 \%$ CI 1.1-1.7) and 1.6 (95\% CI 1.0-2.6). Results were similar after excluding mothers with prepregnancy or pregnancyassociated hypertension, or prepregnancy or gestational diabetes (data not shown).

\section{DISCUSSION}

We hypothesized that in utero conditions associated with maternal BMI-and in particular high BMI-may result in negative effects on fetal development that would then translate to increased susceptibility of developing BPD after delivery. It is thought that multiple inflammatory insults occuring in utero and postnatally may together contribute to the development of BPD (25). Obesity is associated with chronic inflammation in the mother and also possibly the fetus and newborn (17). Using a large population-based data set, we found that high, as well as low, maternal prepregnancy BMI was associated with a modestly increased risk of the development of BPD.

Maternal BMI has been hypothesized to have a wide range of negative impacts on fetal programming and infant and child development $(17,18)$. We are unaware of prior studies examining maternal BMI and BPD. We cannot confirm what mechanisms may contribute to our findings, but inflammation is one possibility for high-BMI patients. Underweight BMI tends to be associated with worse general health status and infant outcomes such as growth retardation and preterm delivery $(19,26,27)$; mechanisms underlying its association with BPD are uncertain but merit further study.

The association of extremes of maternal BMI and BPD were stronger among infants born at 25-29 weeks and among male infants. It is possible that maternal BMI does not contribute to risk among the earliest-born infants, who are also at the highest risk of developing BPD. Alternative explanations include the more limited sample size among these infants and the fact that they have the lowest risk of surviving to 36 weeks and therefore decreased opportunity to have BPD diagnosed at 36 weeks. The stronger findings among male infants are interesting, given that sex-specific adaptations to an adverse uterine environment have been observed, especially with respect to inflammation. Males are more likely than females to be born preterm; one explanation is that the proinflammatory response is greater in male than female pregnancies exposed to infection (28), which in turn is supported by the increased evidence of inflammation in placentas from male than female pregnancies (29). This 
a

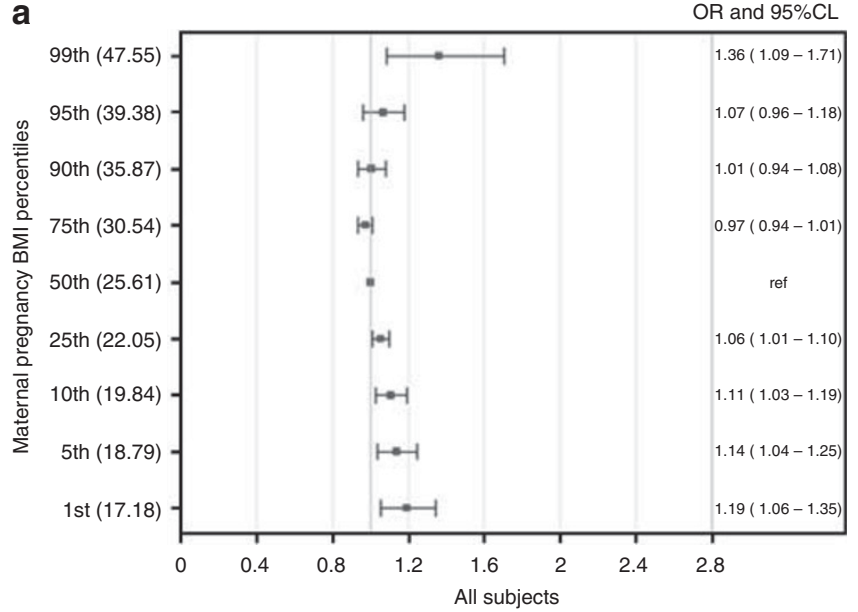

C

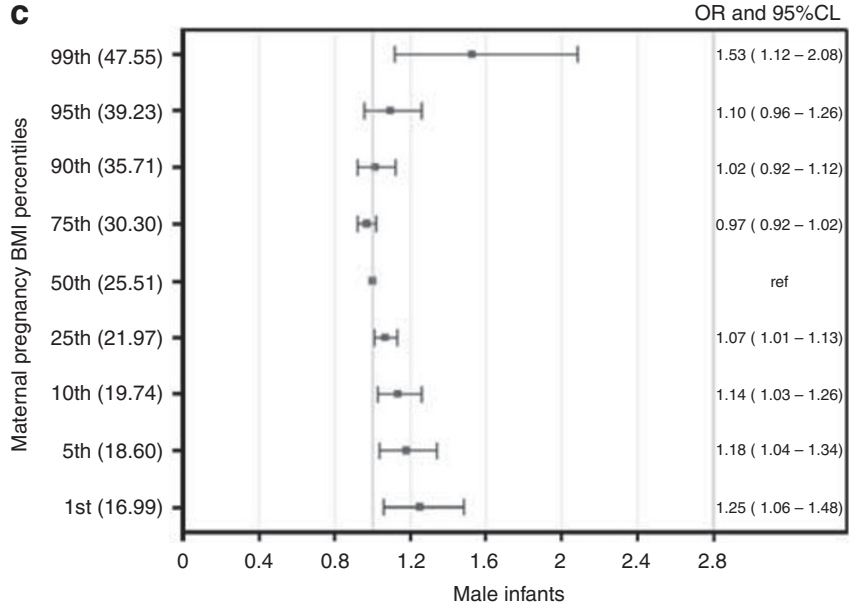

b

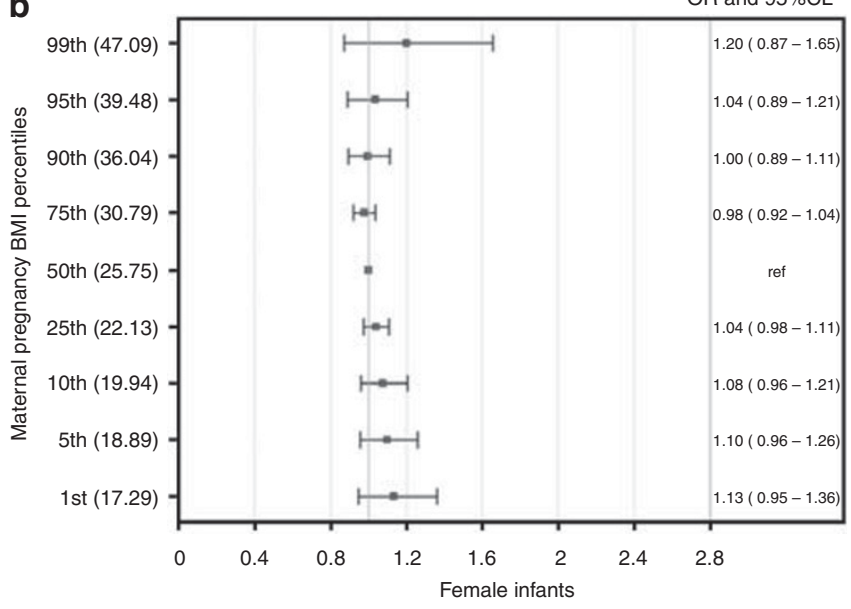

d

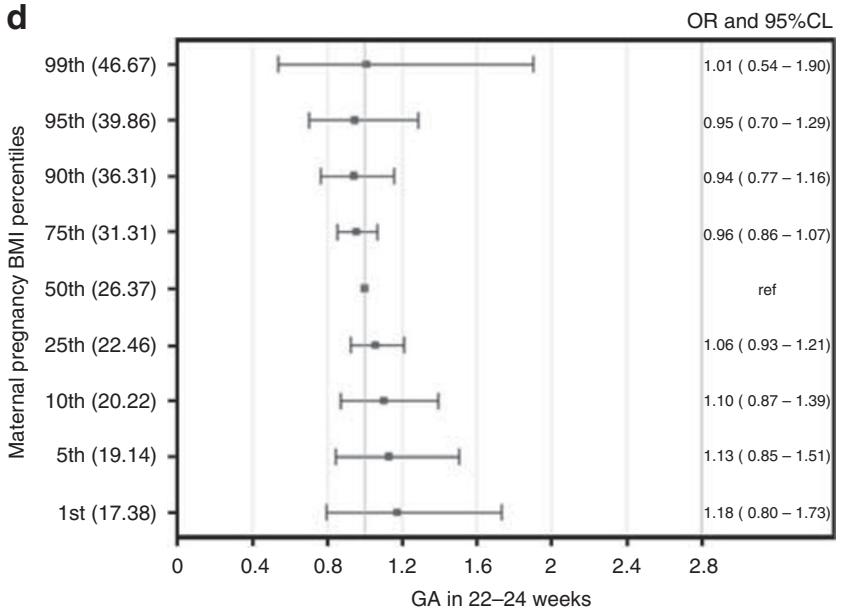

e

OR and $95 \% \mathrm{CL}$

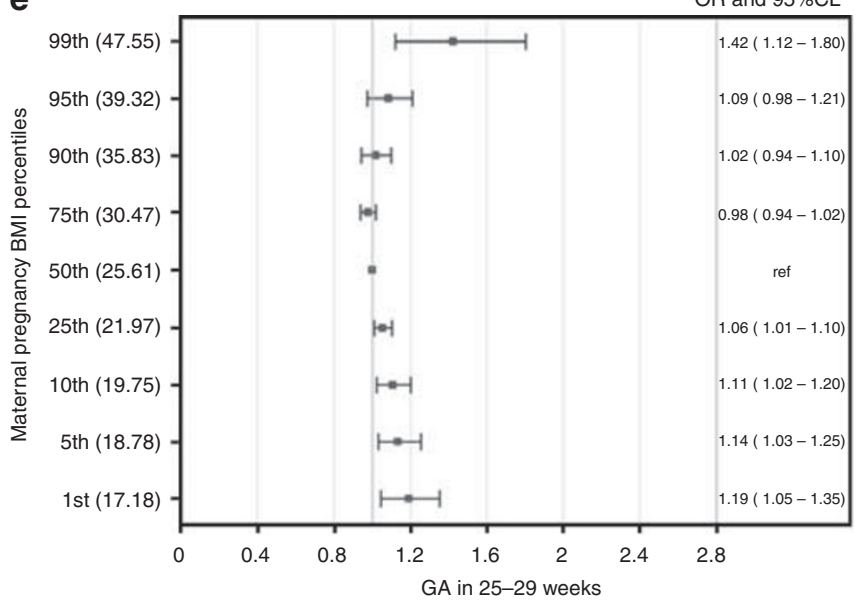

Figure 1. Association of the risk of bronchopulmonary dysplasia with maternal prepregnancy BMI, based on models that included BMI as a continuous variable and BMI-squared (as a test for nonlinearity of association), California, 2007-2011, overall and by infant sex and gestational age at delivery. Models included maternal BMI $\left(\mathrm{kg} / \mathrm{m}^{2}\right)$, BMI-squared, race/ethnicity, age, education, and principal source of payment for delivery, and gestational age and infant sex if not stratified on those variables. (a) All subjects (3,456 cases and 6,726 controls, $P$-value for BMI-squared $<0.01$ ).

(b) Female infants (1,458 cases and 3,259 controls, $P$-value for BMl-squared $=0.11)$. (c) Male infants $(1,998$ cases and 3,467 controls, $P$-value for BMI-squared $<0.01$ ). (d) Infants born at $22-24$ weeks of gestation (808 cases and 347 controls, $P$-value for BMI-squared $=0.53$ ). (e) Infants born at 25-29 weeks of gestation $(2,648$ cases and 6,379 controls, $P$-value for BMI-squared $<0.01)$. 


\section{Articles | Carmichael et al.}

information supports inflammation as a contributor to our findings.

A strength of this study is its population-based design within a racially/ethnically diverse population, which enhances its generalizability. Another strength is the collection of the BPD variable through CPQCC, where clinical data are collected by trained clinical personnel at each center, and the ability to link CPQCC data with vital records to obtain further information about the study subjects such as BMI.

An important limitation is the inability to diagnose BPD before 36 weeks' postmenstrual age (inherent to its definition), combined with substantial mortality (17\%) before this age. If fetal or infant death is associated with maternal BMI and susceptibility to BPD, this could bias results; however, if for example babies born to mothers at the extremes of BMI are more likely to die and to be eventual cases, this would bias results toward the null. We did conduct an analysis that included deaths as cases (which resulted in 5,565 rather than 3,456 cases being included), and results were not substantially different. Our analysis relied on data linkage of clinical and administrative data sets. Infants with unlinked records were substantially more likely than those with linked records to have died ( $55 \%$ vs. $18 \%$ ), have birthweight $<750$ g (51\% vs. $30 \%$ ), and be born at the earliest gestational age of 22 weeks (24\% vs. $4 \%)$. However, unlinked records were similar on other factors (e.g., maternal BMI, age, parity) and comprised only $7 \%$ of all records, and thus we expect that their exclusion did not substantially influence our results. Another limitation of our study was that the duration of oxygen requirement was unknown, and therefore some cases of BPD may have been situations in which babies were on oxygen for acute complications around 36 weeks, but did not have BPD.

In conclusion, we provide evidence that low maternal BMI and high maternal BMI were associated with a modest increased risk of BPD. These findings support the notion that BPD is a multi-factorial disease that in some instances may have its origins in utero and provides some evidence that maternal inflammation may contribute to its development. Although preterm birth is the main risk factor for BPD, the recognition of maternal contributing factors may help us to better develop strategies for understanding its causes and identifying which infants are at risk, and eventual prevention and treatment.

\section{ACKNOWLEDGMENTS}

We thank Hugh O'Brodovich for his mentorship and guidance for this project, including development of the study and editorial feedback.

\section{AUTHOR CONTRIBUTION}

S.L.C. wrote the first draft of the manuscript.

\section{STATEMENT OF FINANCIAL SUPPORT}

This study was supported in part by the March of Dimes Prematurity Research Center at Stanford (MOD PR625253), the Stanford Child Health Research Institute, and by the Stanford Clinical and Translational Science Award (CTSA to Spectrum (UL1 TR001085)).
Disclosure: The authors declare no conflict of interest.

\section{REFERENCES}

1. Thébaud B. Chronic lung disease in the neonate: past, present, and future. NeoReviews 2013;14:e252-8.

2. Northway WH Jr, Rosan RC, Porter DY. Pulmonary disease following respirator therapy of hyaline-membrane disease. Bronchopulmonary dysplasia. N Engl J Med 1967;276:357-68.

3. Murthy K, Porta NF, Lagatta JM, et al. Inter-center variation in death or tracheostomy placement in infants with severe bronchopulmonary dysplasia. J Perinatol 2017 (e-pub ahead of print; doi: 10.1038/ jp.2016.277).

4. Shah PS, Sankaran K, Aziz K, et al. Outcomes of preterm infants $<29$ weeks gestation over 10-year period in Canada: a cause for concern? J Perinatol 2012;32:132-8.

5. Lapcharoensap W, Gage SC, Kan P, et al. Hospital variation and risk factors for bronchopulmonary dysplasia in a population-based cohort. JAMA Pediatr 2015;169:e143676.

6. Bancalari E. Epidemiology and risk factors for the "new" bronchopulmonary dysplasia. NeoReviews 2000;1:e2-5.

7. Gonzalez A, Sosenko IR, Chandar J, Hummler H, Claure N, Bancalari E. Influence of infection on patent ductus arteriosus and chronic lung disease in premature infants weighing 1000 grams or less. J Pediatr 1996;128:470-8.

8. Groneck P, Gotze-Speer B, Oppermann M, Eiffert H, Speer CP. Association of pulmonary inflammation and increased microvascular permeability during the development of bronchopulmonary dysplasia: a sequential analysis of inflammatory mediators in respiratory fluids of high-risk preterm neonates. Pediatrics 1994;93:712-8.

9. Jobe AH. The new BPD. NeoReviews 2006;7:e531-45.

10. Wang EE, Ohlsson A, Kellner JD. Association of Ureaplasma urealyticum colonization with chronic lung disease of prematurity: results of a metaanalysis. J Pediatr 1995;127:640-4.

11. Van Marter LJ, Dammann O, Allred EN, et al. Chorioamnionitis, mechanical ventilation, and postnatal sepsis as modulators of chronic lung disease in preterm infants. J Pediatr 2002;140:171-6.

12. Shaw GM, Wise PH, Mayo J, et al. Maternal prepregnancy body mass index and risk of spontaneous preterm birth. Paediatr Perinat Epidemiol 2014;28:302-11.

13. McDonald SD, Han Z, Mulla S, Beyene J. Overweight and obesity in mothers and risk of preterm birth and low birth weight infants: systematic review and meta-analyses. BMJ 2010;341:c3428.

14. Bhattacharya S, Campbell DM, Liston WA. Effect of body mass index on pregnancy outcomes in nulliparous women delivering singleton babies. BMC Public Health 2007;7:168.

15. Crane JM, Murphy P, Burrage L, Hutchens D. Maternal and perinatal outcomes of extreme obesity in pregnancy. J Obstet Gynaecol Can 2013;35:606-11.

16. Kalk P, Guthmann F, Krause K, et al. Impact of maternal body mass index on neonatal outcome. Eur J Med Res 2009;14:216-2.

17. Rizzo GS, Sen S. Maternal obesity and immune dysregulation in mother and infant: a review of the evidence. Paediatr Resp Rev 2015;16:251-7.

18. Santangeli L, Sattar N, Huda SS. Impact of maternal obesity on perinatal and childhood outcomes. Best Pract Res Clin Obstet Gynaecol 2015;29: 438-8.

19. Girsen AI, Mayo JA, Carmichael SL, et al. Women's prepregnancy underweight as a risk factor for preterm birth: a retrospective study. BJOG 2016;123:2001-7.

20. California Perinatal Quality Care Collaborative (http://cpqcc.org/ about_us). Accessed 1 March 2017.

21. Vermont Oxford Network (http://www.vtoxford.org/about/about.aspx). Accessed 1 March 2017.

22. Gage S, Kan P, Lee HC, et al. Maternal asthma, preterm birth, and risk of bronchopulmonary dysplasia. J Pediatr 2015;167:e1.

23. Oken E, Kleinman KP, Rich-Edwards J, Gillman MW. A nearly continuous measure of birth weight for gestational age using a United States national reference. BMC Pediatr 2003;3:6. 
24. CPQCC Network Database Manual of Definitions for Infants Born in 2010 Version 1.10 (http://cpqcc.org/data/cpqcc_downloads).

25. Jobe AH. Mechanisms of lung injury and bronchopulmonary dysplasia. Am J Perinatol. 2016;33:1076-8.

26. Ali SM, Lindstrom M. Socioeconomic, psychosocial, behavioural, and psychological determinants of BMI among young women: differing patterns for underweight and overweight/obesity. Eur J Public Health 2006;16:325-1.
27. Jeric M, Roje D, Medic N, Strinic T, Mestrovic Z, Vulic M. Maternal prepregnancy underweight and fetal growth in relation to Institute of Medicine recommendations for gestational weight gain. Early Hum Dev 2013;89:277-81.

28. Challis J, Newnham J, Petraglia F, Yeganegi M, Bocking A. Fetal sex and preterm birth. Placenta 2013;34:95-.

29. Clifton VL. Review: sex and the human placenta: mediating differential strategies of fetal growth and survival. Placenta 2010;31 (Suppl): S33-9. 